\title{
Direct observation of van der Waals two-dimensional crystal and small clusters of methane on copper surfaces
}

\author{
Takashi Kumagai, ${ }^{1,2}$ Janina N. Ladenthin, ${ }^{1}$ and Ikutaro Hamada ${ }^{3,4}$ \\ ${ }^{1}$ Department of Physical Chemistry, Fritz-Haber Institute of the Max-Planck Society, Faradayweg 4-6, 14195 Berlin, Germany \\ ${ }^{2}$ JST-PRESTO, 4-1-8 Honcho, Kawaguchi, Saitama 332-0012, Japan \\ ${ }^{3}$ Department of Precision Science and Technology, Graduate School of Engineering, Osaka University, \\ 2-1 Yamada-Oka, Suita, Osaka 565-0871, Japan \\ ${ }^{4}$ Global Research Center for Environment and Energy based on Nanomaterials Science, \\ 1-1 Namiki, Tsukuba, Ibaraki 305-0044, Japan
}

(Received 12 June 2018; published 17 September 2018)

\begin{abstract}
Methane is the simplest hydrocarbon and its aggregation structures are determined merely by van der Waals $(\mathrm{vdW})$ interaction. Here we present the direct observation of a vdW two-dimensional crystal and small clusters of methane on $\mathrm{Cu}$ surfaces using a low-temperature scanning tunneling microscope. Methane shows a hexagonal molecular-packing structure on the $\mathrm{Cu}(110)$ and $\mathrm{Cu}(111)$ surfaces. However, methane preferentially forms a dimer and a trimer on the $\mathrm{Cu}(110)-(2 \times 1)-\mathrm{O}$ added-row structure, indicating different aggregation behavior from the bare $\mathrm{Cu}$ surfaces. The adsorption structures are examined in detail by density functional theory calculations, providing atomistic insights into the adsorption geometry and aggregation mechanism of methane on the $\mathrm{Cu}$ surfaces, which are governed by a subtle balance between the intermolecular and molecule-surface interactions.
\end{abstract}

DOI: 10.1103/PhysRevMaterials.2.093403

Methane is an important chemical feedstock and fuel, which exists in natural gas, shale gas, and ocean floors as methane hydrate [1], and is also related to climate changes [2] and astrochemistry in interstellar media [3]. Methane is also the simplest model of molecular crystals whose aggregation behavior is governed merely by the van der Waals ( $\mathrm{vdW})$ interaction. As being a prototypical molecular crystal, the threedimensional structure and the solid-state phase diagram of methane have been investigated in detail using x-ray/neutron diffraction [4-6], vibrational spectroscopy [7], and theoretical calculations $[8,9]$. Early studies found that a methane crystal forms a face-centered cubic lattice (phase I), indicating that the molecules behave as a simple sphere [10-12]. However, subsequent studies have revealed that methane crystals exhibit a complex phase diagram with at least seven different phases [7]. Furthermore, a methane dimer is routinely employed as a benchmark model of intermolecular interactions in quantum chemical calculations [13]. In experiments small clusters of methane were isolated in cold helium droplets and investigated with high-resolution rotational-vibrational spectroscopy [14]. However, a real-space imaging of single methane molecules remains a challenging experiment. Here we report the direct observation of methane on surfaces using a low-temperature scanning tunneling microscope (STM).

Sample preparation and STM measurements were carried out in ultrahigh vacuum chambers $\left(<5 \times 10^{-10} \mathrm{mbar}\right)$ equipped with a low-temperature STM. An electrochemically etched PtIr tip was used as an STM probe. The bias voltage $\left(V_{\text {bias }}\right)$ was applied to the sample with the tip ground. The tunneling current $\left(I_{\mathrm{t}}\right)$ was corrected from the tip. The $\mathrm{Cu}(110)$ and $\mathrm{Cu}(111)$ surfaces were cleaned by argon-ion sputtering and annealing. Methane $\left(\mathrm{CH}_{4}\right)$ was adsorbed by backfilling the STM chamber. Our density functional theory (DFT) calculations were performed by using a plane-wave basis and pseudopotential method as implemented in the STATE code [15]. We used the rev-vdW-DF2 [16] exchangecorrelation functional with an efficient implementation [17,18] (see Supplemental Material [19] for further details of the calculations).

Figure 1(a) shows an STM image of the $\mathrm{Cu}(110)$ surface exposed to a 22 Langmuir $\left(1 \mathrm{~L}=1 \times 10^{-6}\right.$ Torr s) methane gas at $\sim 13 \mathrm{~K}$. Small protrusions on the surface correspond to single methane molecules or small clusters. The molecules diffused on the surface even at $5 \mathrm{~K}$, suggesting that methane is weakly bound to the surface and the adsorption potential is expected to be very shallow. As the methane dose was increased $(\sim 80 \mathrm{~L})$, the molecules aggregated into a twodimensional island on the surface [Fig. 1(b)]. The islands were formed around a single-atomic step of the surface, which implies that the growth occurs from the step edges. The individual methane molecules in the island could be resolved as a round protrusion in the high-resolution STM image [Fig. 1(c)]. It should be noted that the high-resolution image was obtained most probably by a functionalized tip with a methane molecule [20] that was occasionally picked up to the tip apex at the island edge.

We found that methane forms a hexagonal molecularpacking structure with a lattice constant of $4.2( \pm 0.1) \AA$ [Fig. 1(d)]. This distance coincides with the carbon-carbon distance of the methane crystal in the face-centered cubic structure (phase I) $[11,21]$. Thus, the rectangular $\left(C_{2 v}\right.$ symmetry) atomic arrangement of the $\mathrm{Cu}(110)$ surface is not reflected in the two-dimensional structure, suggesting that the aggregation is dominated by the $\mathrm{vdW}$ interaction between 
(a)

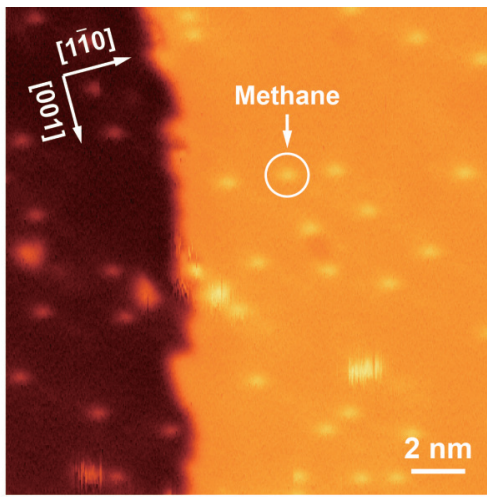

(b)

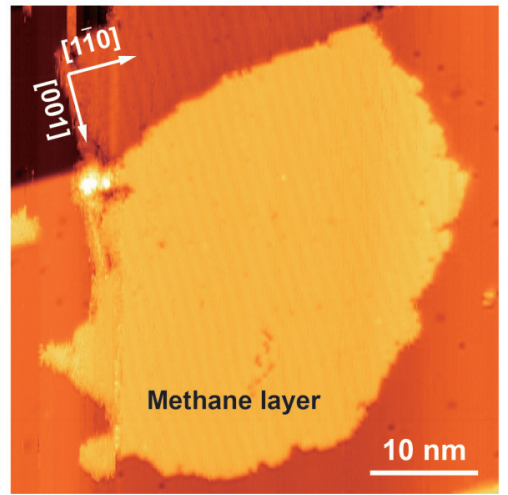

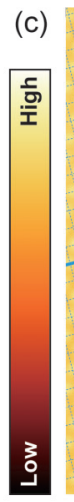

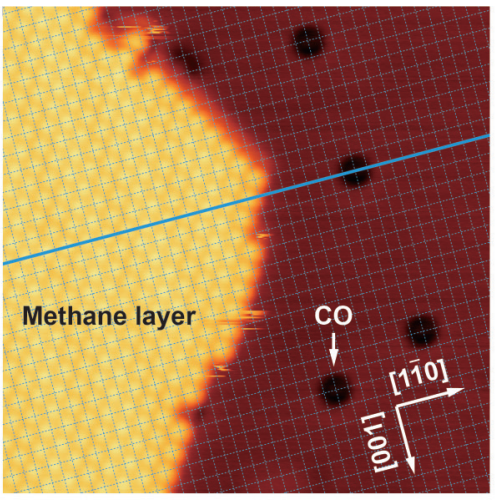

(e)

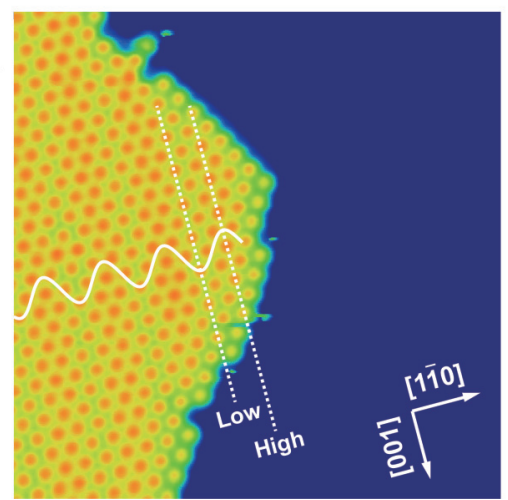

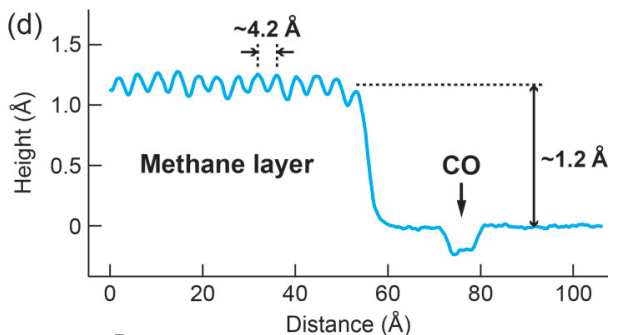

(f) $\stackrel{2}{2}$

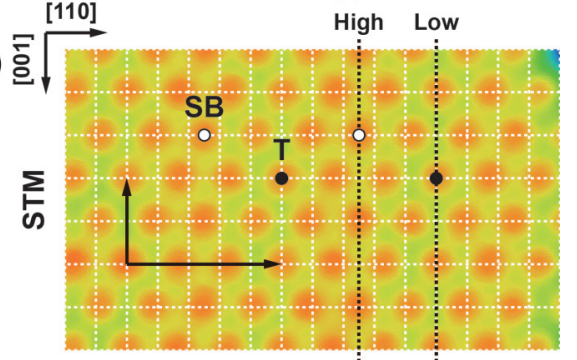

(g)

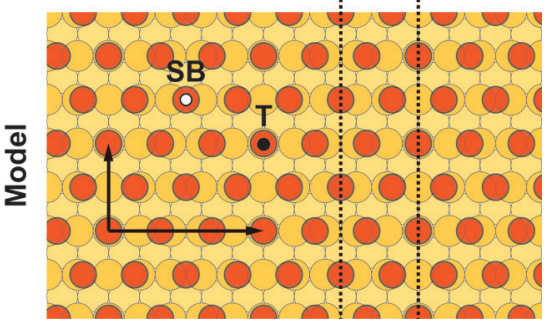

FIG. 1. (a) STM image of methane molecules on $\mathrm{Cu}(110)$ at low coverage $\left(5 \mathrm{~K}, V_{\text {bias }}=20 \mathrm{mV} ; I_{\mathrm{t}}=5 \mathrm{pA}\right)$. (b) Overview STM image of a methane island on $\mathrm{Cu}(110)\left(5 \mathrm{~K}, V_{\text {bias }}=30 \mathrm{mV} ; I_{\mathrm{t}}=20 \mathrm{pA}\right)$. (c) High-resolution STM image of a methane island on $\mathrm{Cu}(110)(5 \mathrm{~K}$, $\left.V_{\text {bias }}=30 \mathrm{mV} ; I_{\mathrm{t}}=50 \mathrm{pA}\right)$. CO molecules were coadsorbed as a marker to identify the adsorption position of the methane molecules with respect to the $\mathrm{Cu}(110)$ lattice (represented by the blue grid lines). (d) Line profile along the blue solid line in (c) where a methane layer and a CO molecule are observed. (e) Same STM image as (c) in a different color scale to emphasize the Moiré pattern in the methane layer. The white waved line represents the Moiré corrugation to guide the eye. (f) Enlarged STM image of the methane layer. The white grid lines represent the surface lattice of $\mathrm{Cu}(110)$. The white and black dots indicate the short-bridge (SB) and the on-top (T) sites, respectively. The black arrows indicate the $\mathrm{CH}_{4}-(3 \times 2) / \mathrm{Cu}(111)-(5 \times 2)$ supercell. The dashed black lines represent the highest and lowest topographic height in the Moiré corrugation to guide the eye. $(\mathrm{g})$ Schematic model of the methane layer on $\mathrm{Cu}(110)$. The red circles indicate the adsorption site of the methane molecules.

methane molecules, whereas the molecule-surface interaction plays a minor role to determine the lateral geometry. However, the absence of a three-dimensional growth of methane islands indicates that the methane-surface interaction is stronger than that of methane with its first layer.

The STM image of the methane layer shows a subtle Moiré pattern [Fig. 1(e)] resulting from a lattice mismatch with the surface. In order to reveal the adsorption site of individual molecules, the surface lattice of $\mathrm{Cu}(110)$ was determined by coadsorbing $\mathrm{CO}$ molecules on the surface. A CO molecule adsorbs onto the on-top site of $\mathrm{Cu}(110)$ [22] and can be identified by STM inelastic electron tunneling spectroscopy [23], thus acting as a good positional marker. The grid lines in Figs. 1(c) and 1(f) represent the surface lattice of $\mathrm{Cu}(110)$, showing that the methane molecules adsorb on the $\mathrm{Cu}$ atomic

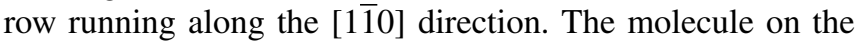
short-bridge (SB) and on-top (T) site exhibits the highest and lowest topographic height, respectively [Fig. 1(f)]. However, since the STM image corresponds to the local density of states of the sample surface, it is not straightforward to directly correlate the apparent height with the actual geometric height of methane on the surface. In addition, as mentioned above, the tip apex was terminated by a methane molecule, which may affect the apparent height. Therefore, we avoid quantitative discussion about the geometric height of the methane molecules. However, the lateral dimensions can be determined and the schematic adsorption geometry of the methane layer is shown in Fig. 1(g).

Figure 2(a) shows a methane island formed on a $\mathrm{Cu}(111)$ surface obtained by exposure to a $20 \mathrm{~L}$ methane gas at $\sim 13 \mathrm{~K}$. In common with a methane layer on $\mathrm{Cu}(110)$, the molecules form a hexagonal molecular-packing structure on $\mathrm{Cu}(111)$ with a lattice constant of $4.3( \pm 0.1) \AA$. This result verifies that the two-dimensional structure [Fig. 2(b)] of methane is not significantly influenced by the atomic arrangement of the $\mathrm{Cu}$ surfaces. However, the Moiré pattern is not clearly visible on $\mathrm{Cu}(111)$ because it overlaps with a modulated local density of states caused by the interference of the surface state [24].

Figures 3(a) and 3(b) show the optimized adsorption structure of a single methane molecule on the $\mathrm{T}$ and $\mathrm{SB}$ site of $\mathrm{Cu}(110)$, respectively, obtained by the DFT calculations. We examined various adsorption geometries and found comparable total energies for several different structures as expected for the very weak interaction between methane and the surface 
(a)

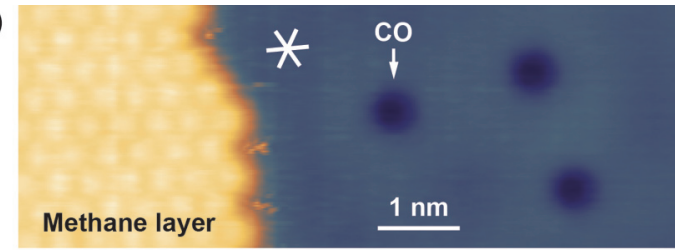

(b)

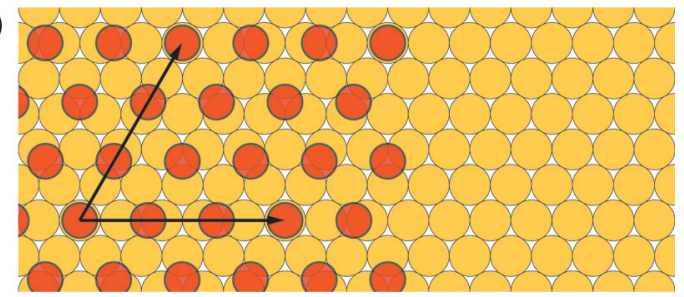

FIG. 2. Methane on $\mathrm{Cu}(111)$. (a) High resolution STM image of a methane layer on $\mathrm{Cu}(111)\left(5 \mathrm{~K}, V_{\text {bias }}=30 \mathrm{mV} ; I_{\mathrm{t}}=50 \mathrm{pA}\right) . \mathrm{CO}$ molecules were co-adsorbed as a marker to identify the adsorption position of the methane molecules with respect to the $\mathrm{Cu}(111)$ lattice. The white lines in the image represent the high-symmetry axes of the surface. (b) Structure model of the methane molecules (red circles) on $\mathrm{Cu}(111)$. The black arrows indicate the $\mathrm{CH}_{4}-(3 \times$ $3) / \mathrm{Cu}(111)-(5 \times 5)$ supercell.

(see Supplemental Material [19]). It was revealed that the molecular orientation with the three $\mathrm{C}-\mathrm{H}$ legs down to the surface (3-leg configuration) is more stable than the others (1- or 2-leg configuration). However, methane may freely rotate on the adsorption site even at low temperatures as observed in methane crystals [25]. Figure 3(c) shows the optimized structure of the methane monolayer on $\mathrm{Cu}(110)$ with the 3-leg configuration (see Supplemental Material [19] for the 1-leg and 2-leg configurations). The average inter-
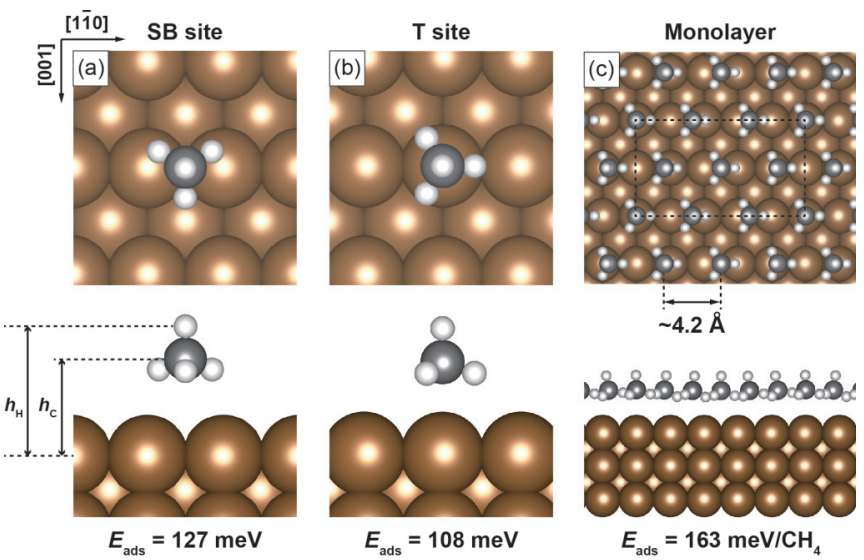

FIG. 3. Calculated structures of a single methane molecule on $\mathrm{Cu}(110)$ : On the short-bridge (SB) site (a) and on the on-top (T) site (b). The height of $\mathrm{C}$ atom $\left(h_{\mathrm{C}}\right)$ (apical $\mathrm{H}$ atom, $h_{\mathrm{H}}$ ) of methane is 3.25 (4.34) $\AA$ and 2.98 (4.08) $\AA$ for the SB and T sites, respectively. The height is defined as the distance from the center of carbon atom to the average position of the center of $\mathrm{Cu}$ atoms in the topmost layer. (c) Methane monolayer on $\mathrm{Cu}(110)$. The black dashed box indicate the cell of the $\mathrm{CH}_{4}-(3 \times 2) / \mathrm{Cu}(111)-(5 \times 2)$ supercell. The average $\mathrm{C}$ (apical $\mathrm{H}$ ) height is 3.22 (4.31) $\AA$, and the maximum and the minimum heights are 3.26 (4.36) $\AA$ and 3.19 (4.29) $\AA$ at $\mathrm{T}$ and SB sites, respectively.
TABLE I. Calculated adsorption energy $\left(E_{\text {ads }}\right)$ of the methane monolayer on $\mathrm{Cu}(110)$, defined by $E_{\text {ads }}=-\frac{1}{n}\left[E_{\text {tot }}\left\{\left(\mathrm{CH}_{4}\right)_{n} /\right.\right.$ $\left.\mathrm{Cu}(110)\}-n E_{\text {tot }}\left\{\mathrm{CH}_{4}\right\}-E_{\text {tot }}\{\mathrm{Cu}(110)\}\right]$, where $E_{\text {tot }}\left\{\left(\mathrm{CH}_{4}\right)_{n} /\right.$ $\mathrm{Cu}(110)\}, E_{\text {tot }}\left\{\mathrm{CH}_{4}\right\}$, and $E_{\text {tot }}\{\mathrm{Cu}(110)\}$ are the total energies of the $\mathrm{CH}_{4}$ monolayer on $\mathrm{Cu}(110)$, isolated $\mathrm{CH}_{4}$, and the $\mathrm{Cu}(110)$ substrate, respectively, and $n$ is the number of $\mathrm{CH}_{4}$ molecules. $E_{\text {ads }}^{\text {mol-surf }}$ and $E_{\text {ads }}^{\text {mol-mol }}$ represent the $\mathrm{CH}_{4}$-surface and intermolecular adsorption energies, respectively (see Supplemental Material [19] for the definitions). A positive value indicates the adsorption state is more stable than the isolated ones. The nonlocal correlation contribution to the adsorption energy is given in the parenthesis.

\begin{tabular}{ccc}
\hline \hline$E_{\text {ads }}\left(\mathrm{meV} / \mathrm{CH}_{4}\right)$ & $E_{\text {ads }}^{\text {mol-surf }}\left(\mathrm{meV} / \mathrm{CH}_{4}\right)$ & $E_{\text {ads }}^{\text {mol-mol }}\left(\mathrm{meV} / \mathrm{CH}_{4}\right)$ \\
\hline $163(302)$ & $120(222)$ & $42(80)$ \\
\hline \hline
\end{tabular}

molecular distance (carbon-carbon) of about $4.2 \AA$ is in excellent agreement with experiment. Table I lists the adsorption energy, the molecule-surface, and intermolecular interaction energies of the methane monolayer on the $\mathrm{Cu}(110)$ surface along with the contribution from the nonlocal correlation, which is responsible for the vdW forces (see Supplemental Material [19] for the definition). It was found that the nonlocal correlation contribution is dominant in the adsorption energy, and the calculations clearly indicate that the molecule-surface and intermolecular vdW interactions stabilize the monolayer structure. The height of the carbon atom in the isolated methane molecule is calculated to be 3.25 and $2.98 \AA$ for the SB and $\mathrm{T}$ sites, respectively. On the other hand, the highest and lowest heights of methane in the monolayer are found at the T (3.26 $\AA$ ) and SB sites (3.19 $\AA$ ), thus the vdW interactions between molecules affect the geometric height. We also performed constant-current STM simulations based on the Tersoff-Hamann theory [26,27], and found that the apparent height on the T site is higher than the SB site. This result appears to be inconsistent with the topographic STM image in Fig. 1(f). However, the difference calculated for the methane height on the T and SB sites $(0.07 \AA)$ is rather small and it is not trivial to compare with the topographic height in the STM image (the influence of a methane-terminated tip is also unclear, as mentioned above).

We found that the aggregation behavior of methane considerably differs on the $\mathrm{Cu}(110)-(2 \times 1)-\mathrm{O}$ added-row structure. This reconstruction occurs by adsorption of oxygen on the clean $\mathrm{Cu}(110)$ surface and alternating $\mathrm{Cu}$ and $\mathrm{O}$ chains grow in the [001] direction [28,29]. Figure 4(a) displays an overview image of the surface partially covered with the $\mathrm{Cu}(110)-(2 \times 1)-\mathrm{O}$ structure, which is exposed to a $20-\mathrm{L}$ methane gas at $\sim 13 \mathrm{~K}$. The bare $\mathrm{Cu}(110)-(2 \times 1)-\mathrm{O}$ structures appear as characteristic dark stripes running along the [001] direction and methane is observed as bright protrusions. The molecules preferentially adsorbed onto the $\mathrm{Cu}(110)-(2 \times$ 1)-O structure and no molecule was observed on the bare $\mathrm{Cu}$ surface at a low coverage, indicating that the methane-surface interaction is stronger than the bare surface. Figure 4(b) shows a high-resolution image of methane on the $\mathrm{Cu}(110)-(2 \times$ 1)-O structure. The molecules aggregate into a dimer or a trimer along the added-row as indicated by the red and blue dashed box in Fig. 4(b). Additionally, they form a column-like structure along the [110] direction as indicated by the white 
(a)

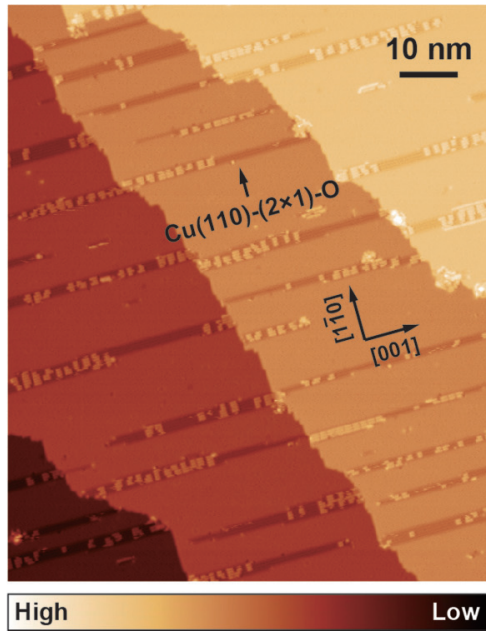

(b)

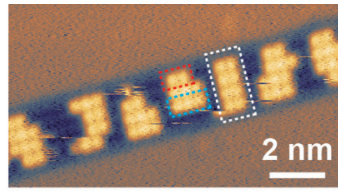

(c)

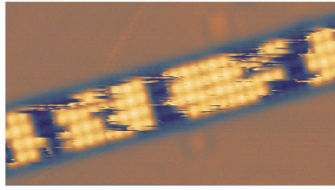

(d)

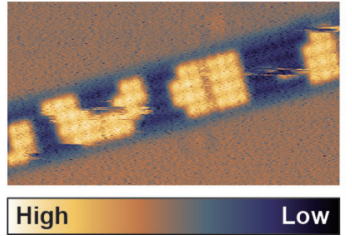

(e)

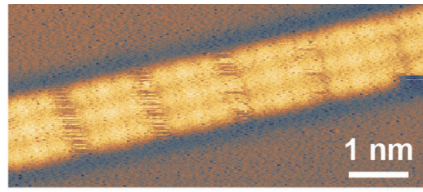

(f)

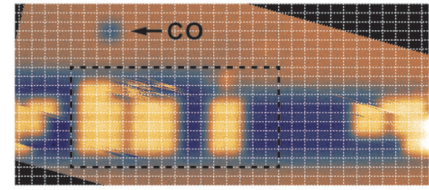

(g) 1000000000-030

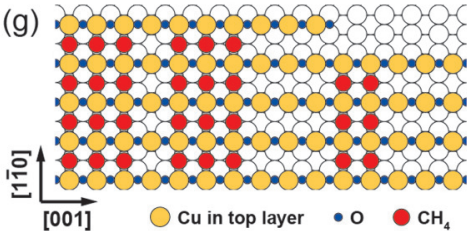

FIG. 4. Methane adsorption on the $\mathrm{Cu}(110)-(2 \times 1)$-O added-row structure. (a) Overview STM image of the surface exposed to a methane gas $\left(5 \mathrm{~K}, V_{\text {bias }}=20 \mathrm{mV}, I_{\mathrm{t}}=3 \mathrm{pA}\right)$. (b)-(d) Sequential STM images of methane molecules on the added-row structure [ $5 \mathrm{~K}$, (b) $V_{\text {bias }}=20 \mathrm{mV}$, $I_{\mathrm{t}}=3 \mathrm{pA}$; (c) $V_{\text {bias }}=20 \mathrm{mV}, I_{\mathrm{t}}=10 \mathrm{pA}$; (d) $V_{\text {bias }}=20 \mathrm{mV}, I_{\mathrm{t}}=3 \mathrm{pA}$. The red and blue dashed boxes in (b) indicate a dimer and a trimer unit along the [001] direction. The white dashed box in (b) indicates the column-like aggregation along [110] direction. (e) STM image of methane molecules on the added-row structure at a high coverage $\left(5 \mathrm{~K}, V_{\text {bias }}=20 \mathrm{mV}, I_{\mathrm{t}}=3 \mathrm{pA}\right)$. (f) STM image of methane molecules on the added-row structure with a CO marker $\left(5 \mathrm{~K}, V_{\text {bias }}=20 \mathrm{mV}, I_{\mathrm{t}}=3 \mathrm{pA}\right)$. The white grid lines represent the surface lattice of Cu(110). (g) Structure model for the area indicated by the black dashed box in (f).

dashed box in Fig. 4(b). The intermolecular distance of the methane clusters is about $3.6 \AA$ which is smaller than that of the two-dimensional methane layer on the bare $\mathrm{Cu}$ surfaces as well as the three-dimensional methane crystal $(\sim 4.2 \AA)$. This result demonstrates a crucial impact of the substrate on the aggregation behavior of methane.

The stationary image of methane could be obtained under very moderate scanning conditions such as $V_{\text {bias }}=20 \mathrm{mV}$ and $I_{\mathrm{t}}=3 \mathrm{pA}$ and the molecules diffused along the added-row structure when the $V_{\text {bias }}$ and/or $I_{\mathrm{t}}$ were slightly increased [Fig. 4(c)], suggesting that the methane-surface interaction is again relatively weak. However, methane remained as the dimer and the trimer and the column-like structure was also kept even after STM-induced diffusion [Fig. 4(d)]. Figure 4(e) displays methane on the added-row structure at a higher coverage, where the molecules aggregate into trimers along the added-row and form a periodic structure. The adsorption site of methane is determined using a CO marker [Fig. 4(f)]. We found that the molecules are located in the trough of the $\mathrm{Cu}(110)-(2 \times 1)-\mathrm{O}$ structure and adsorb at the long-bridge site between the $\mathrm{Cu}$ atoms [Fig. $4(\mathrm{~g})$ ].

Figure 5(a) shows the most stable structure of a single methane molecule on the $\mathrm{Cu}(110)-(2 \times 1)-\mathrm{O}$ structure calculated by DFT. The methane molecule at the long-bridge site between the $\mathrm{Cu}$ atoms is more favorable than the other sites (see Supplemental Material [19]), in good agreement with the experiment. As is the case in methane on the clean $\mathrm{Cu}(110)$ surface, the molecule favors the 3-leg conformation compared to 1- and 2-leg ones. The adsorption energy of methane on the $\mathrm{Cu}(110)-(2 \times 1)-\mathrm{O}$ structure is significantly larger than those on the bare $\mathrm{Cu}(110)$ surface, which may be explained by the more ionic nature of the former structure. The charge density
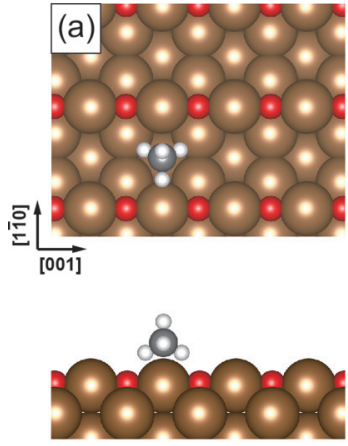

$E_{\text {ads }}=145 \mathrm{meV} / \mathrm{CH}_{4}$
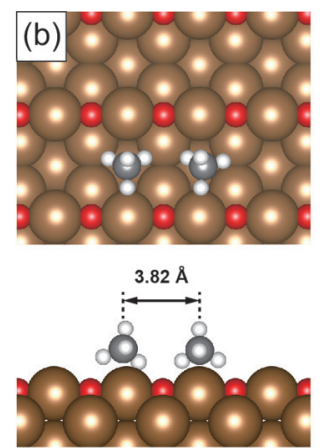

$E_{\text {ads }}=153{\mathrm{meV} / \mathrm{CH}_{4}}$
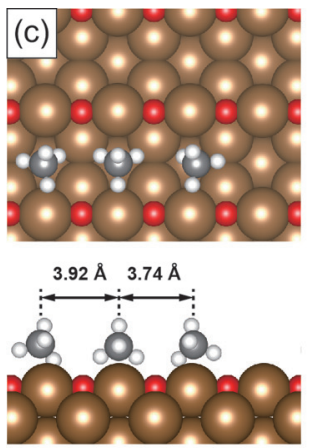

$E_{\text {ads }}=160 \mathrm{mV} / \mathrm{CH}_{4}$

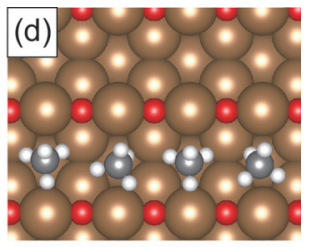

$3.68 \AA 3.54 \AA 3.53 \AA$

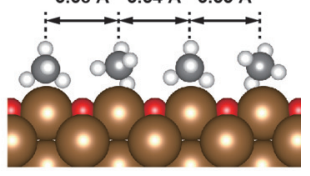

$E_{\text {ads }}=146 \mathrm{meV} / \mathrm{CH}_{4}$

FIG. 5. Calculated structure of methane on the $\mathrm{Cu}(110)-(2 \times 1)-\mathrm{O}$ added-row structure. (a) Monomer $\left(h_{\mathrm{C}}: 2.09 \AA\right)$. (b) Dimer $\left(h_{\mathrm{C}}: 2.18\right.$ and $2.10 \AA$ from the left). (c) Trimer $\left(h_{\mathrm{C}}: 2.26,2.14\right.$, and $2.22 \AA$ from the left). (d) Tetramer $\left(h_{\mathrm{C}}: 2.15,2.33,2.21\right.$, and $2.29 \AA$ from the left). The height $\left(h_{\mathrm{C}}\right)$ is defined as the distance from the center of carbon atom to the average position of between the $\mathrm{Cu}$ and $\mathrm{O}$ atoms at the topmost layer. 
TABLE II. Calculated adsorption energy $\left(E_{\mathrm{ads}}\right)$ of the methane monomer and clusters on the $\mathrm{Cu}(110)-(2 \times 1)-\mathrm{O}$ structure, defined by $E_{\text {ads }}=-\frac{1}{n}\left[E_{\text {tot }}\left\{\left(\mathrm{CH}_{4}\right)_{n} / \mathrm{CuO}\right\}-n E_{\text {tot }}\left\{\mathrm{CH}_{4}\right\}-E_{\text {tot }}\{\mathrm{CuO}\}\right]$, where $E_{\text {tot }}\left\{\left(\mathrm{CH}_{4}\right)_{n} / \mathrm{CuO}\right\}, E_{\text {tot }}\left\{\mathrm{CH}_{4}\right\}$, and $E_{\text {tot }}\{\mathrm{CuO}\}$ are the total energies of the $\mathrm{CH}_{4}$ molecule(s) on the $\mathrm{Cu}(110)-(2 \times 1)-\mathrm{O}$ added-row structure, isolated $\mathrm{CH}_{4}$, and the $\mathrm{Cu}(110)$ substrate including the $\mathrm{Cu}(110)-(2 \times 1)-\mathrm{O}$ added-row structure, respectively, and $n$ is the number of the $\mathrm{CH}_{4}$ molecules. $E_{\text {ads }}^{\mathrm{mol}-\text { surf }}$ and $E_{\text {ads }}^{\mathrm{mol}-\mathrm{mol}}$ represent the $\mathrm{CH}_{4}$-surface and intermolecular adsorption energies, respectively. The nonlocal correlation contribution to the adsorption energy is given in the parenthesis.

\begin{tabular}{lcccc}
\hline \hline & Monomer & Dimer & Trimer & Tetramer \\
\hline$E_{\text {ads }}\left(\mathrm{meV} / \mathrm{CH}_{4}\right)$ & $145(322)$ & $153(301)$ & $160(297)$ & $146(320)$ \\
$E_{\text {ads }}^{\text {molsurf }}\left(\mathrm{meV} / \mathrm{CH}_{4}\right)$ & - & $155(282)$ & $158(271)$ & $160(270)$ \\
$E_{\text {ads }}^{\text {mol-mol }}\left(\mathrm{meV} / \mathrm{CH}_{4}\right)$ & - & $-1(19)$ & $2(26)$ & $-14(50)$ \\
\hline \hline
\end{tabular}

of the $\mathrm{Cu}(110)-(2 \times 1)-\mathrm{O}$ surface is more polarized than that of the $\mathrm{Cu}(110)$ surface, in particular, in the vicinity of $\mathrm{O}$ atoms, suggesting that the polarization of the surface enhances the adsorption of methane (see Supplemental Material [19]).

The calculations predicted that the dimer [Fig. 5(b)] and the trimer [Fig. 5(c)] are slightly more stable than the monomer. As shown in Table I, the adsorption energies of the dimer and the trimer are larger than the monomer on the $\mathrm{Cu}(110)-(2 \times 1)-\mathrm{O}$ structure and the bare $\mathrm{Cu}(110)$ surface. The stabilization is attributed to the methane-surface interaction. On the other hand, the tetramer [Fig. 5(d)] shows the smaller adsorption energy (Table II) than the dimer and the trimer, consistent with the experiment (absence of tetramer). The calculations revealed that the intermolecular distance subtly varies depending on the cluster size, although it is not discernible in the STM image. The calculated intermolecular distance of 3.7-3.9 $\AA$ in the dimer and the trimer is smaller than that in the methane layer on the $\mathrm{Cu}(110)$ surfaces and in the bulk crystal. In contrast to the methane layer, the intermolecular interaction of the dimer and the trimer does not contribute to the structure stabilization and the slightly larger adsorption energy than the monomer on the $\mathrm{Cu}(110)-(2 \times 1)-\mathrm{O}$ structure may be attributed to a substratemediated interaction. However, the intermolecular distances in the tetramer are forced to be even smaller than those in the dimer and the trimer because structural relaxation of the molecules is more restricted. This situation causes repulsive intermolecular interactions and eventually leads to destabilization of the tetramer. The column-like structure along the [11̄0] direction observed in the experiment (Fig. 4) suggests a substrate-mediated interaction between methane clusters. However, the calculations did not find a clear stabilization mechanism across the $\mathrm{O}$-added row.

In summary, we directly observed a two-dimensional crystal and small clusters of methane on $\mathrm{Cu}$ surfaces with a low-temperature STM. Our combined experimental and theoretical study showed how the subtle intermolecular and molecule-substrate interactions through $\mathrm{vdW}$ forces determine the aggregation behavior. It was found that methane forms the hexagonal molecular-packing structure on the $\mathrm{Cu}(110)$ and $\mathrm{Cu}(111)$ surfaces with the intermolecular distance of $\sim 4.2 \AA$, which is almost identical to that of the bulk crystal. On the $\mathrm{Cu}(110)-(2 \times 1)-\mathrm{O}$ structure, methane formed a dimer and a trimer along the trough of the oxygenadded rows but larger clusters were not observed. Our DFT simulations showed excellent agreement with experiments and provided atomistic insights into the adsorption structures and the aggregation mechanism. We found that methane prefers to adsorb with the three $\mathrm{C}-\mathrm{H}$ bonds downward to the surface and the methane monolayer on the $\mathrm{Cu}(110)$ surface is stabilized through vdW interactions among the molecules. However, the methane-surface interaction becomes stronger on the $\mathrm{Cu}(110)-(2 \times 1)-\mathrm{O}$ structure due to its more ionic nature. It was revealed that the dimer and the trimer are stabilized by the molecule-surface interaction, whereas there is almost no energy gain through the intermolecular interaction because of a shorter distance between the molecules than the equilibrium distance. In the tetramer, the intermolecular distances become even shorter, leading to destabilization through repulsive forces between the molecules. The aggregation behavior and structure on surfaces are essentially related to methane conversion with heterogeneous catalysis. Our approach will pave the way for investigating elementary surface processes of methane in real space.

The authors thank Jun Yoshinobu for careful reading of the manuscript. T.K. acknowledges the support by JST-PRESTO (JPMJPR16S6) and the Deutsche Forschungsgemeinschaft through Sfb951. I.H. acknowledges the support by MEXT Program for Development of Environmental Technology using Nanotechnology. The numerical calculations were performed using the Numerical Materials Simulator at National Institute for Materials Science, Japan.
[1] K. A. Kvenvolden, Methane hydrate - A major reservoir of carbon in the shallow geosphere? Chem. Geol. 71, 41 (1988).

[2] C. D. Ruppel and J. D. Kessler, The interaction of climate change and methane hydrates, Rev. Geophys. 55, 126 (2017).

[3] J. H. Lacy, J. S. Carr, N. J. Evans II, F. Baas, J. M. Achtermann, and J. F. Arens, Discovery of interstellar methane - Observations of gaseous and solid $\mathrm{CH}_{4}$ absorption toward young stars in molecular clouds, Astrophys. J. 376, 556 (1991).

[4] M. A. Neumann, W. Press, C. Noldeke, B. Asmussen, M. Prager, and R. M. Ibberson, The crystal structure of methane phase III, J. Chem. Phys. 119, 1586 (2003).
[5] H. E. Maynard-Casely, C. L. Bull, M. Guthrie, I. Loa, M. I. McMahon, E. Gregoryanz, R. J. Nelmes, and J. S. Loveday, The distorted close-packed crystal structure of methane A, J. Chem. Phys. 133, 064504 (2010).

[6] H. E. Maynard-Casely, L. F. Lundegaard, I. Loa, M. I. McMahon, E. Gregoryanz, R. J. Nelmes, and J. S. Loveday, The crystal structure of methane $\mathrm{B}$ at $8 \mathrm{GPa}-\mathrm{An} \alpha-\mathrm{Mn}$ arrangement of molecules, J. Chem. Phys. 141, 234313 (2014).

[7] R. Bini and G. Pratesi, High-pressure infrared study of solid methane: Phase diagram up to $30 \mathrm{GPa}$, Phys. Rev. B 55, 14800 (1997). 
[8] T. Yamamoto and Y. Kataoka, Quantum Statistical Mechanical Study on Phase Transitions in Solid Methane, Phys. Rev. Lett. 20, 1 (1968).

[9] H. Lin, Y.-1. Li, Z. Zeng, X.-j. Chen, and H. Q. Lin, Structural, electronic, and dynamical properties of methane under high pressure, J. Chem. Phys. 134, 064515 (2011).

[10] J. C. Mclennan and W. G. Plummer, The crystal structure of solid methane, Philos. Mag. 7, 761 (1929).

[11] H. H. Mooy, Crystal structure of methane, Nature (London) 127, 707 (1931).

[12] A. Müller and A. Schaliamach, Crystal structure of methane at the transition point, $20.4^{\circ} \mathrm{K}$, Nature (London) 143, 375 (1939).

[13] A. H.-T. Li and S. D. Chao, Interaction energies of dispersionbound methane dimer from coupled cluster method at complete basis set limit, J. Mol. Struct. Theochem. 897, 90 (2009).

[14] H. Hoshina, D. Skvortsov, M. N. Slipchenko, B. G. Sartakov, and A. F. Vilesov, Rotation of methane molecules in dimers and small clusters, J. Chem. Phys. 143, 084305 (2015).

[15] Y. Morikawa, H. Ishii, and K. Seki, Theoretical study of $n$ alkane adsorption on metal surfaces, Phys. Rev. B 69, 041403 (2004).

[16] I. Hamada, van der Waals density functional made accurate, Phys. Rev. B 89, 121103 (2014).

[17] J. Wu and F. Gygi, A simplified implementation of van der Waals density functionals for first-principles molecular dynamics applications, J. Chem. Phys. 136, 224107 (2012).

[18] Y. Hamamoto, I. Hamada, K. Inagaki, and Y. Morikawa, Selfconsistent van der Waals density functional study of benzene adsorption on Si(100), Phys. Rev. B 93, 245440 (2016).

[19] See Supplemental Material at http://link.aps.org/supplemental/ 10.1103/PhysRevMaterials.2.093403 for the details of DFT calculations and additional simulation results.
[20] G. Kichin, C. Weiss, C. Wagner, F. S. Tautz, and R. Temirov, Single molecule and single atom sensors for atomic resolution imaging of chemically complex surfaces, J. Am. Chem. Soc. 133, 16847 (2011).

[21] W. Press, Structure and phase transitions of solid heavy methane $\left(\mathrm{CD}_{4}\right)$, J. Chem. Phys. 56, 2597 (1972).

[22] M. Doering, J. Buisset, H.-P. Rust, B. G. Briner, and A. M. Bradshaw, Adsorption site determination with scanning tunnelling microscopy, Faraday Discuss. 105, 163 (1996).

[23] L. J. Lauhon and W. Ho, Single-molecule vibrational spectroscopy and microscopy: $\mathrm{CO}$ on $\mathrm{Cu}(001)$ and $\mathrm{Cu}(110)$, Phys. Rev. B 60, R8525 (1999).

[24] M. F. Crommie, C. P. Lutz, and D. M. Eigler, Imaging standing waves in a two-dimensional electron gas, Nature (London) 363, 524 (1993).

[25] C. Chapados and A. Cabana, Infrared-spectra and structures of solid $\mathrm{CH}_{4}$ and $\mathrm{CD}_{4}$ in phases I and II, Can. J. Chem. 50, 3521 (1972).

[26] J. Tersoff and D. R. Hamann, Theory and Application for the Scanning Tunneling Microscope, Phys. Rev. Lett. 50, 1998 (1983).

[27] J. Tersoff and D. R. Hamann, Theory of the scanning tunneling microscope, Phys. Rev. B 31, 805 (1985).

[28] F. Jensen, F. Besenbacher, E. Lægsgaard, and I. Stensgaard, Surface reconstruction of $\mathrm{Cu}(110)$ induced by oxygen chemisorption, Phys. Rev. B 41, 10233 (1990).

[29] D. J. Coulman, J. Wintterlin, R. J. Behm, and G. Ertl, Novel Mechanism for the Formation of Chemisorption Phases: The $(2 \times 1) \mathrm{O}-\mathrm{Cu}(110)$ "Added Row" Reconstruction, Phys. Rev. Lett. 64, 1761 (1990). 